\title{
Diagnostic of FET PET in Patients with Recurrent Glioma: Case Series Habibollah Dadgar*
}

RAZAVI Cancer Research Center, RAZAVI Hospital, Imam Reza International University, Mashhad, Iran

*Corresponding author: Habibollah Dadgar, RAZAVI Cancer Research Center, RAZAVI Hospital, Imam Reza International University, Mashhad, Iran, E-mail: reza.lt.dadgar@gmail.com

Received date: August 06, 2018; Accepted date: September 17, 2018; Published date: September 25, 2018

Copyright: $@ 2018$ Dadgar $\mathrm{H}$. This is an open-access article distributed under the terms of the Creative Commons Attribution License, which permits unrestricted use, distribution, and reproduction in any medium, provided the original author and source are credited.

\begin{abstract}
Background: The precise definition margin of high and low-grade gliomas is crucial for treatment. We aimed to assess the feasibility of assessment of the resection legions with post-operative positron emission tomography (PET) using [ $\left.{ }^{18} \mathrm{~F}\right] \mathrm{O}-\left(2-\left[{ }^{18} \mathrm{~F}\right]-\right.$ fluoroethyl)-L-tyrosine $\left(\left[{ }^{18} \mathrm{~F}\right] \mathrm{FET}\right)$.
\end{abstract}

Methods: Four patients with the suspicion of high and low-grade were enrolled. Patients underwent postoperative $\left[{ }^{18} \mathrm{~F}\right]$ FET-PET, pre-operative magnetic resonance imaging (MRI) and CT for clinical evaluations.

Results: In our study, three patients had negative response to recurrence and progression and one patient indicated positive response after surgery. [ $\left.{ }^{18} \mathrm{~F}\right] \mathrm{FET}$-PET revealeda legion of increased radiotracer uptake in the dura in the carniotomy site for patient 1. Corresponding to the patient history, the study was negative for recurrence of brain tumor. For patient 2, There was a lesion in the right parieto-temporal with slightly increased uptake in its posterior part with $S_{U} U V_{m a x}=3.79$, so the study was negative for recurrence evaluation. In patient 3 therewas no abnormal uptake withnegative result for recurrence of brain tumor.Intense radiotracer uptake in the left parietal lobe where in the MRI there was a lesion with no change in enhancement in the post-contrast image is indicated in patient 4 .

Conclusion: Assessment of the resection legions in high and low-grade gliomas with [ $\left.{ }^{18} \mathrm{~F}\right]$ FET-PET seems to be useful. Labelled amino acid tracers may predict the response to the chemo-radiotherapy and early detection of residual tumor after surgery.

Keywords: FET-PETCT; Glioma; MRI; Residual tumor

\section{Introduction}

Prognosis brain tumors in high and low grades using conventional modalities is complex and sophisticated with poor resultant of final data. Meanwhile, MRI is the gold standard for assessment of malignant gliomas and shows high spatial resolution for diagnosis of brain tumors [1,2].

Conventional modalities such as CT and MRI cannot differentiate tumoral tissue from non-neoplastic changes such as oedema, postoperative changes and radiation necrosis. Radio-labelled amino acid imaging is superior to FDG-PET in confirming low-grade recurrence. These days, amino acids labeled radioactive molecules like ${ }^{18} \mathrm{~F}$ can be useful following radiation therapy and surgery. O-(2fluoroethyl)-L-tyrosine has most interest by scientists to measure treatment response in gliomas [3].

Lower uptake of radioactivity in normal brain tissue is due to the higher uptake of amino acid tracers in the tumor receptors which over express in the tumoral cells. Using of amino acids in the grading is controversial while FDG PET appears better differentiated of tumor grades with lower power for differentiates the gliomas from histologically benign brain tumors or non-neoplastic lesions.

Increased amino acid uptake in gliomas which has been observed in non-enhancing tumor regions is difficult to delineate using MRI [4]. This issue indicates that the margins of growth tumor may be underestimated using MRI [5,6]. Moreover, radio-necrosis due to irradiation of tumorhas usually negativeresponse on ${ }^{18} \mathrm{~F}$-FET PET reports [7].

For these reasons, there are clinical evidences for processing $\left[{ }^{18} \mathrm{~F}\right]-$ FET PET data combination with MRI for better interpretation of images [8-10]. Thus, ${ }^{18} \mathrm{~F}$-FET PET may be a promising tool to following the treatment response of patient.

Shortly, FET uptake in the brain tumors or recurrence lesions after surgery can indicate amino acid rates and its accumulation in the cell because this radiotracer is incorporated into proteins and metabolized process after transport to the cell. Moreover, FET has lower uptake in inflammatory cells.

This series report discussabout ${ }^{18}$ F-FET PET analysis of four patients involved low and high grade glioma that followed in the Nuclear Medicine Department, RAZAVI Hospital, Mashhad, Iran.

\section{Materials and Methods}

All patients had concurrence to injection of ${ }^{18} \mathrm{~F}-\mathrm{FET}$ and Subsequently PET/CT imaging protocols was approved by RAZAVI Hospital, Nuclear Medicine Department in 2016.

\section{MRI protocol}

Patients underwent MRI performed on a 1.5 Tesla scanner (Sonata, Siemens-Erlangen, Germany). Sequence protocols such as T2-weighted 
and fast-spin echo sequences $(6600 \mathrm{~ms} / 100-110 \mathrm{~ms}, 2 \mathrm{~mm}$ slice thickness, 3206240 matrixes), fluid attenuated inversion recovery (FLAIR) sequence and T1-weighted gradient-echo sequences (TR=1860 ms, TE=4.38 ms with $1.2 \mathrm{~mm}$ slice thickness, 2566192 matrix) before and after administration of gadolinium was used.

\section{FET-PET protocol}

20 minutes after IV injection of $7.3 \mathrm{mCi}(200-250 \mathrm{MBq}){ }^{18} \mathrm{~F}-\mathrm{FET}$ at 20 minutes image was obtained from the brain using a PET/CT (Biograph) scanner (Siemens Medical Systems, Erlangen, Germany) according to a standardized acquisition protocol.

Semi-quantitative PET data included the maximal standardized uptake value (SUV) of the tumor compared to the background activity in the contralateral hemisphere. A threshold of 1.8 was considered for assessment of GBM.

An iterative reconstruction was obtained using the ordered subset expectation maximization (OSEM) algorithm. Scans were reported with Nuclear Medicine specialists for investigationthe results of ${ }^{18} \mathrm{~F}$ FET PET and the histo-pathological diagnosis.

\section{Results}

Four patients underwent craniotomy to remove a metastatic tumor. These patients first presented to us for imaging to investigate recurrence after 3-4 months of tumor surgery.

\section{Case 1}

This case was a 40-year-old Male with brain tumor, Anaplastic Oligodendroglioma, WHO grade 3. Gross examination showed SRF consist of multiple fragments of creamy soft tissue TM $2 \times 2 \times 0.5 \mathrm{~cm}^{3}$. Moreover, Microscopic Examination dedicated that sections show fragments of neoplastic lesion composed of polygonal or round cells hypochromatic and pleomorphic nuclei clear cytoplasm, high mitotic index, atypical mitotic Figures surrounded by thin wall blood vessels with chicken wire pattern.

MRI of the brain with and without injection (First study): According to previous craniotomy surgery, mild dilatation of the lateral ventricles, injury in the right upper anterior region and Linear Abnormal Signal Intensities in pre-ventrical region no symptoms of recurrence in the right hemisphere is not visible.

MRI of the brain with and without injection (Second study): Changes related to surgery in the right frontal bone and areas adjacent gliosis induced signal change can be seen. High absorption cannot be seen in this area while enhancement in two small nodules in the left frontal lobe was seen. Hyper signal pre-ventrical in some regions in right parietal lobe and left region of frontal corpus callosum, adjacent the left ventricle, are visible. Finally there was no abnormality in the Brain Stem.

FET-PET/CT findings: 20 minutes after IV injection of $7.3 \mathrm{mCi}{ }^{18} \mathrm{~F}$ FET a 20 minutes image was obtained from the brain. Images were reviewed on the monitor's screen. There is a zone of increased radiotracer uptake in the dura in the carniotomy site. There is no site of increased uptake in the brain parenchyma. Site of previous disease is noted in the fronto-parietal area of the right side. The study is negative for recurrence of brain tumor (Figures 1 and 2).

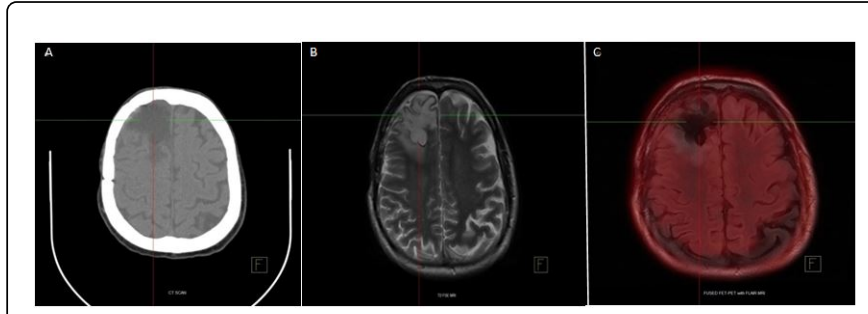

Figure 1: Axial sections of CT, MRI and Fused FET/MRI. A: Shows CT scan after surgery, B: T2-Weithed MRI, C: Fused FET-PET with MRI.

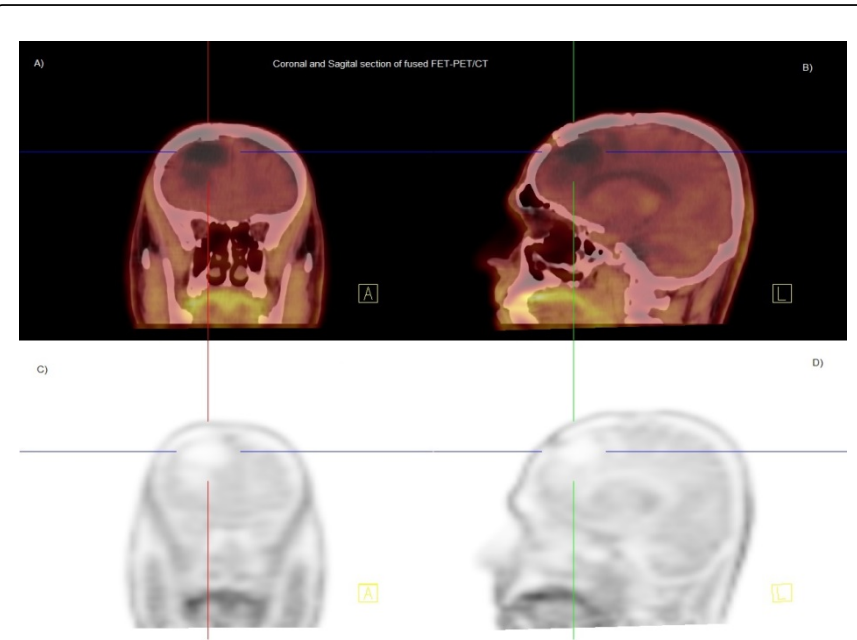

Figure 2: PET and Fused PET/CT. A,C: shows the coronal section of fused PET/CT and FET-PET alone respectively. B,D: indicates the sagittal section of fused PET/CT and FET-PET alone respectively.

\section{Case 2}

This case was 51-year-old Female with brain tumor, Glioblastoma Multiforme. Gross examination showed SRF consist of multiple small fragments of creamy soft tissue and blood clots TM $3 \times 2 \times 1 \mathrm{~cm}^{3}$. Microscopic Examination demonstrated that sections show fragments of brain tissue with astrocytic neoplastic lesion composed of proliferation of atypical glial cells with nuclear atypia and mitotic Figures beside vascular proliferation, endothelial hyperplasia and foci of necrosis.

First MRI study-2014: MRI of the brain in sagittal, coronal and axial sections using T1W, T2W and FLAIR sequences was done and previous craniotom defect in the right parietal bone was seen. In right parietal lobe a mass with approximately dimension of $40 \times 45 \times 48$ $\mathrm{mm}^{3}$ was seen. There was no evidence of defect in cerebellum and medulla oblongata as well and parenchyma of the brain had normal signal intensity.

Second MRI study-2014: MRI of the brain in sagittal, coronal and axial sections using T1W, T2W and FLAIR sequences was done and there was a ensphalomallasi mass in the right temporal with gliosis around it. After the intravenous injection of contrast agent, peripheral enhancement was seen in the lesion but was not in benefit of tumor tissue. 
Third MRI study-2015: MRI of the brain in sagittal, coronal and axial sections using T1W, T2W and FLAIR sequences was done and craniotom defect in the right parietal bone was seen. In right parietal lobe a mass with approximately dimension of $30 \times 52 \mathrm{~mm}^{2}$ was seen. In T1W without contrast agents signal intensity in the peripheral of the lesion was seen that can be due to the existence of calcification. Moreover, after injection contrast agent, there was no enhancement in the mentioned area.

Forth MRI study-2016: MRI of the brain in sagittal, coronal and axial sections using T1W, T2W and FLAIR sequences were done and defects in right parietal bone were shown. In the right parietal lobe, a mass with heterogeneous signal containing solid and cystic areas and approximately dimensions $35 \times 40 \times 45 \mathrm{~mm}^{3}$ was seen that spread from the cortex to lateral wall of the right ventricle. Signal intensity in T1W without contrast agents around the mass can be due to the existence of calcification.

After injection the contrast agent there was no significant enhancement in the mentioned mass. Areas of increased signal intensity in the white matter around the ventricles and also around right parietal lobe respect to radiotherapy can be ensphalopaty due to post-irradiation. On the other hand, there is evidence that indicated mild Ventricolomegally in the ventricles.

FET-PET/CT findings: 20 minutes after IV injection of $7.3 \mathrm{mCi}{ }^{18} \mathrm{~F}$ FET a 20 minutes image was obtained from the brain. Images were reviewed on the monitor's screen. There is a zone of calcified lesion in the right parieto-temporal lesion in the CT part which shows slightly increased uptake in its posterior part with $\mathrm{SUV}_{\max }=3.79$ with 2.68 in the contralateral part (1.4 times more uptake).The study is negative for recurrence or residual disease in the right parieto-temporal lobe (Figure 3).

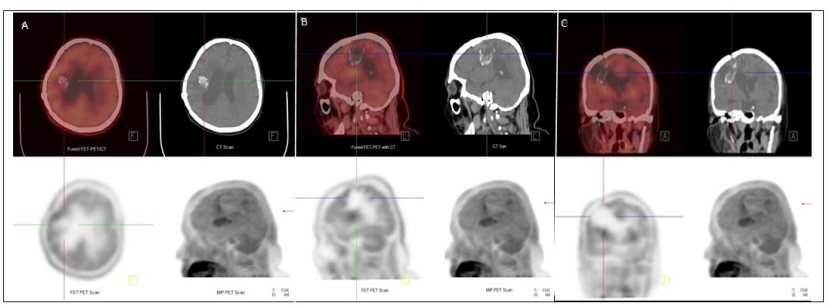

Figure 3: Axial, Sagittal and Coronal sections of negative recurrence response to FET-PET after surgery.

\section{Case 3}

This case was 73-year-old Male with volume loss in left frontal lobe. MRI; 2016. There is an evidence of malacic changes and volume loss in left frontal lobe. After GAD injection, pachymeningeal enhancement is visualized over left cerebral convexity and left para-midline falx. No nodular enhancement is seen. Bilateral symmetric superatentorialvasogenic edema is visualized which might be due to treatment effect.

Moderate volume loss of cerebral white matter is compatible with senile ischemic changes. Complete opacification and mucosal thickening of left maxillary sinus is seen. Control PNS CT is recommended. Otherwise study seems unremarkable. Clinical correlation and comparison with previous studies are helpful.
FET-PET/CT findings: 20 minutes after IV injection of $7.3 \mathrm{mCi}{ }^{18} \mathrm{~F}-$ FET at 20 minutes image was obtained from the brain. Images were reviewed on the monitor's screen. There is No zone of abnormal increased radiotracer uptake in the brain tissue. There is a calcification in the left frontal area in the CT part which shows no abnormal uptake. The study is negative for recurrence of brain tumor (Figure 4).

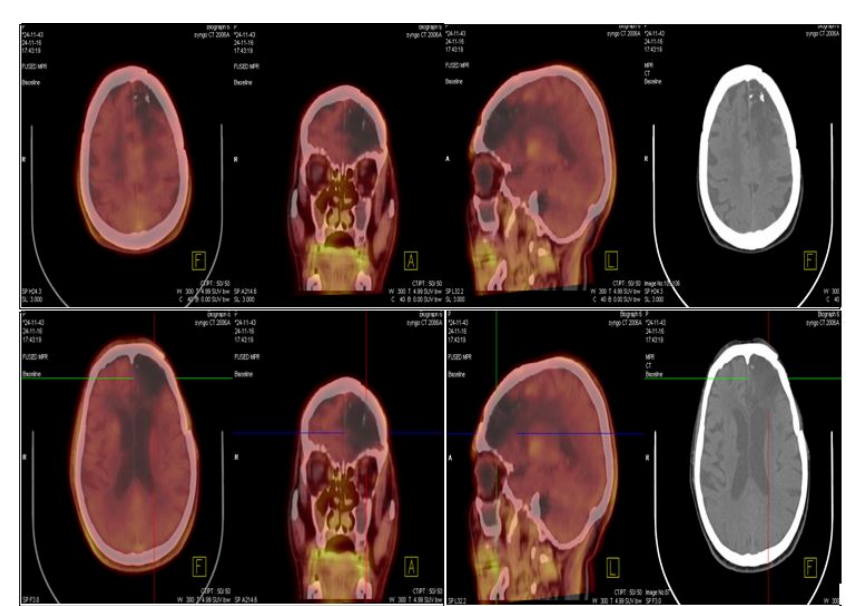

Figure 4: Axial and Coronal sections of negative recurrence response to FET-PET after surgery.

\section{Case 4}

This case was 38-year-old Male with brain tumor, low grade glioma consistent with oligodendroglioma, WHO grade 2. Clinical data showed generalized seizure resistant to medical treatment and a left parietal lobe mass without enhancement. Macroscopic Examination showed Specimen consists of multiple tiny pieces of creamy brown soft tissue embedded in blood clot TM $5 \times 10 \times 20 \mathrm{~mm}$. In Microscopic Examination sections showed white and gray matter brain tissue involved by neoplasm with moderate cellularity composed of cells with round nuclie and clear perinuclear clear halos admixed with some minigemistocyts. Preneuronalsatellitosis and mild nuclear pleomorphism are also seen. No evidence of mitotic activity, vascular endotelial proliferation or necrosis is identified.

FET-PET/CT findings: 20 minutes after IV injection of $7.3 \mathrm{mCi}{ }^{18} \mathrm{~F}$ FET a 20 minutes image was obtained from the brain. Images were reviewed on the monitor's screen. There is intense radiotracer uptake in the left parietal lobe where in the MRI there is a lesion with no change in enhancement in the post-contrast image. $\mathrm{SUV}_{\max }$ of the lesion is 7.68 and corresponding contra lateral region shows $\mathrm{SUV}_{\max }=$ 1.8 which makes its uptake around 4.3 times more. Recurrence or residual disease is noted in the left parietal lobe (Figure 5). 


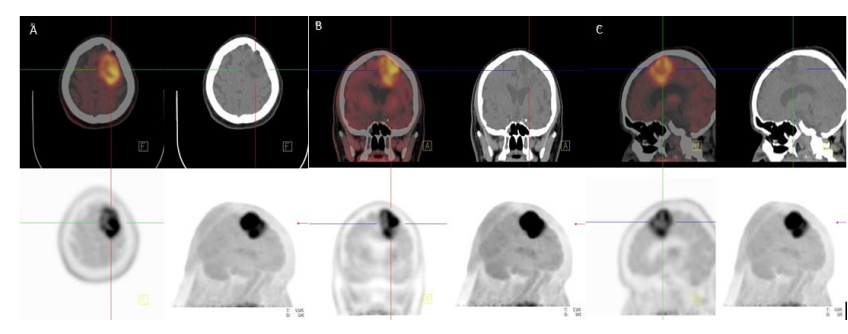

Figure 5: A,B,C: Axial, Coronal and Sagittal section of CT, FET-PET only and Fused FET-PET/CT for positive tumor recurrence monitoring.

\section{Discussion}

Since conventional imaging, MRI and CT, are unable to distinguish between necrosis of radiation therapy and tumor recurrence, it is very important to overcome to this problem. Meanwhile, FET-PET is a powerful tool to differentiate between post-operative lesions and tumor recurrence and also grading of the brain tumors [11-13]. The results of our study were in agreement with several ${ }^{18} \mathrm{~F}$-FETstudies for tumor recurrence evaluation $[14,15]$. CT and MRI could not show functional response for delineation of recurrence tumor after craniotomy to remove a metastatic tumor (Anaplastic Oligodendroglioma, WHO grade 3) in frontal lobe in patient 1, furthermore functional imaging like amino acidligand conjugated ${ }^{18} \mathrm{~F}$ may be useful for assessment of the tumor margins for following the surgery (Figure 1). As it is shown in Figure 2 there was no site of increased uptake in the brain parenchyma. So according to the MRI and also FET-PET results, the study was negative for recurrence of brain tumor. Moreover, because CT and MRI are not gold standards for evaluation of recurrence and grading tumor after surgery, physicians would like to have more information about tumor response to the local craniotomy and therapy. In Figure 3 tumor bed is clearly revealed in $\mathrm{CT}$ and accordingly functional FET-PET illustrated that the study was negative for recurrence or residual disease in the right parieto-temporal lobe. In Figure 4 there was intense radiotracer uptake in the left parietal lobe where in the MRI there is a lesion with no change in enhancement in the post-contrast image. Recurrence or residual disease was noted in the left parietal lobe. In short, combinations of FET-PET and MRI havehigh sensitivity for evaluation of recurrence and grading of the brain tumors. These results have a good agreement with study of Dunet et al. [16] They used dynamic FET PET for initial glioma grading for 38 patients and then compared it to MRI findings. Therefore, the use of FET-PET/MR may be helpful for assessment of brain tumors.

\section{Following}

After following patients who enrolled in current study, one of them who had positive response to FET scan obtained 60 days chemotherapy using temozolomide. Others did not need any therapeutic for their disease due to the negative response FET.

\section{Conclusion}

The current study indicates that FET PET has significantly high diagnostic performance for diagnosis of brain tumor and grading of gliomas. FET-PET can distinguish between tumoral and non-tumoral lesions and is superior for brain lesion characterization. Overall sensitivity of ${ }^{18}$ F-FET for glioma monitoring was higher than MRI and CT. In short, labeled amino acid may predict the response to the chemo-radiotherapy and early detection of residual tumor after surgery.

\section{References}

1. De Angelis LM (2001) Brain tumors. N Engl J Med 344: 114-123.

2. Kumar AJ, Leeds NE, Fuller GN, Van Tassel P, Maor MH, et al. (2000) Malignant gliomas: MR imaging spectrumof radiation therapy and chemotherapy-induced necrosis of the brain aftertreatment. Radiology 217: 377-384.

3. Jager PL, VaalburgW, Pruim J, de Vries EG, Langen KJ, et al. (2001) Radiolabeledamino acids: Basic aspects and clinical applications in oncology. J Nucl Med 42: 432-445.

4. Pauleit D, Floeth F, Hamacher K, Riemenschneider MJ, Reifenberger G, et al. (2005) O-(2-[18F]fluoroethyl)-L-tyrosine PETcombined with MRI improves the diagnostic assessment of cerebral gliomas. Brain 128: 678-687.

5. Galldiks N, Ullrich R, Schroeter M, Fink GR, Kracht LW (2010) Volumetry of $[(11) \mathrm{C}]$-methionine PET uptake and MRI contrast enhancement in patients withrecurrent glioblastomamultiforme. Eur J Nucl Med Mol Imaging 37: 84-92.

6. Grosu AL, Astner ST, Riedel E, Nieder C, Wiedenmann N, et al. (2011) An interindividual comparison of O-(2- [(18)F]Fluoroethyl)-L-Tyrosine (FET)- andL-[Methyl-(11)C]Methionine (MET)-PET in patients with brain gliomas and metastases. Int J Radiat Oncol Biol Phys 81: 1049-1058.

7. Po“pperl G, Go“tz C, Rachinger W, Gildehaus FJ, Tonn JC (2004) Value of $\mathrm{O}-(2-[18 \mathrm{~F}]$ fluoroethyl)-L-tyrosine PET for the diagnosis of recurrent glioma. Eur J Nucl Med Mol Imaging 31: 1464-1470.

8. Pirotte BJ, Levivier M, Goldman S, Massager N, Wikler D, et al. (2009) Positronemission tomography-guided volumetric resection of supratentorial high-gradegliomas: a survival analysis in 66 consecutive patients. Neurosurgery 64: 471-481

9. Weber DC, Zilli T, Buchegger F, Casanova N, Haller G, et al. (2008) [(18)F] Fluoroethyltyrosine-positron emission tomography-guided radiotherapyfor high-grade glioma. RadiatOncol 3: 44.

10. Plotkin M, Blechschmidt C, Auf G, Nyuyki F, Geworski L, et al. (2010) Comparison of F-18 FET-PET with F-18 FDG-PET for biopsy planning of noncontrast-enhancing gliomas. Eur Radiol 20: 2496-2502.

11. Rachinger W, Goetz C, Popperl (2005) Positron emission tomography with O-(2-[18F]fluoroethyl)-L-tyrosine versus magnetic resonance imaging in thediagnosis of recurrent gliomas. Neurosurgery 57: 505-511.

12. Bertolini F, Bagni B, Valentini A (2008) 18F-FET PET in early detection of relapse/progression in high-grade gliomas. Neuro Oncol 10: 1099-1100.

13. Brandes AA, Tosoni A, Spagnolli F (2008) Disease progression orpseudoprogression after concomitant radiochemotherapy treatment: Pitfalls in neurooncology. Neuro Oncol 10: 361-367.

14. Herholz K, Kracht LW, Heiss WD (2003) Monitoring the effect of chemotherapy ina mixed glioma by C-11-methionine PET. J Neuroimaging 13: 269-271.

15. Hamacher K, Coenen $\mathrm{HH}$ (2002) Efficient routine production of the $18 \mathrm{~F}$ labelled aminoacid O-2-18F fluoroethyl-L-tyrosine. Appl Radiat Isot 57: 853-856.

16. Dunet V, Maeder P, Nicod-Lalonde M, Lhermitte B, Pollo C, et al. (2014) Combination of MRI and dynamic FET PET for initial glioma grading. Nklearmedizin 53: 155-161. 